\title{
Anomalous behaviour of surface Brillouin scattering in thin strained CVD diamond
}

DOI:

10.1016/j.diamond.2020.108020

\section{Document Version}

Accepted author manuscript

Link to publication record in Manchester Research Explorer

\section{Citation for published version (APA):}

Aradi, E. (2020). Anomalous behaviour of surface Brillouin scattering in thin strained CVD diamond. Diamond and Related Materials. https://doi.org/10.1016/j.diamond.2020.108020

\section{Published in:}

Diamond and Related Materials

\section{Citing this paper}

Please note that where the full-text provided on Manchester Research Explorer is the Author Accepted Manuscript or Proof version this may differ from the final Published version. If citing, it is advised that you check and use the publisher's definitive version.

\section{General rights}

Copyright and moral rights for the publications made accessible in the Research Explorer are retained by the authors and/or other copyright owners and it is a condition of accessing publications that users recognise and abide by the legal requirements associated with these rights.

\section{Takedown policy}

If you believe that this document breaches copyright please refer to the University of Manchester's Takedown Procedures [http://man.ac.uk/04Y6Bo] or contact uml.scholarlycommunications@manchester.ac.uk providing relevant details, so we can investigate your claim.

\section{OPEN ACCESS}




\section{Journal Pre-proof}

Anomalous behaviour of surface Brillouin scattering in thin strained CVD diamond

I. Motochi, B.A. Mathe, S.R. Naidoo, E. Aradi

PII:

S0925-9635(20)30573-2

DOI:

https://doi.org/10.1016/j.diamond.2020.108020

Reference:

DIAMAT 108020

To appear in:

Diamond \& Related Materials

Received date:

15 January 2020

Revised date:

26 July 2020

Accepted date:

29 July 2020

Please cite this article as: I. Motochi, B.A. Mathe, S.R. Naidoo, et al., Anomalous behaviour of surface Brillouin scattering in thin strained CVD diamond, Diamond \& Related Materials (2020), https://doi.org/10.1016/j.diamond.2020.108020

This is a PDF file of an article that has undergone enhancements after acceptance, such as the addition of a cover page and metadata, and formatting for readability, but it is not yet the definitive version of record. This version will undergo additional copyediting, typesetting and review before it is published in its final form, but we are providing this version to give early visibility of the article. Please note that, during the production process, errors may be discovered which could affect the content, and all legal disclaimers that apply to the journal pertain.

(C) 2020 Published by Elsevier. 


\title{
Anomalous behavior of surface Brillouin scattering in thin strained CVD diamond
}

\author{
I. Motochi ${ }^{* 1}$, B. A. Mathe ${ }^{2}$, S. R. Naidoo ${ }^{2}$, E. Aradi ${ }^{3}$ \\ ${ }^{1}$ Dept of Mathematics and Physical Sciences, Maasai Mara University, P.O Box 861-20500, \\ Narok, Kenya Motochi@mmarau.ac.ke \\ ${ }^{2}$ Materials Research Institute, DST-NRF Centre of Excellence in Strong Materials and School of \\ Physics, University of the Witwatersrand, Wits 2050, Johu.'nesburg, S. Africa. \\ ${ }^{3}$ School of Computing and Engineering, University of Hudder, fiels, Queensgate, Huddersfield \\ HD1 3DH, United Kingdo. ๆ
}

July 26,2020

\begin{abstract}
A bs. ract
Hellium ions were implanted into diamond . + nergies of 80 and $160 \mathrm{keV}$ with fluences beyond the amorphization threshold using the Cola 'mplantation Rapid Annealing (CIRA) technique. A thin recrystallized diamond layer of $\approx 63 \mathrm{n}^{\mathrm{r}} \mathrm{l}$ ad $\approx 103 \mathrm{~nm}$ for implantation at $80 \mathrm{keV}$ and $160 \mathrm{keV}$, respectively was estimated using t! $\mathrm{e}$ c $\mathrm{nstant}$ angle reflection interference spectroscopy. Analysis on this thin diamond-graphitic cá hon-bulk diamond structure was carried out using Raman spectroscopy, diffuse reflecta. e spectroscopy and surface Brillouin scattering. Raman analysis shows that the damage ${ }^{\prime} b$ - rie $\AA$ layer consists of an amorphous graphitic material while the diffuse reflectance measuremerts showed high reflectance at the diamond-graphitic layer interface. This led to multiple reflectivity resulting in reduction of the intensity of the incident beam to $\approx 0.37$ that gets reflected. Surface Brillouin scattering showed a weak longitudinal peak, $\mathrm{L}_{K B 2}$ at $61.6 \mathrm{GHz}$ for the recrystallized diamond layer while for the unimplanted diamond a similar peak was observed at $64.2 \mathrm{GHz}$ for laser beam $\lambda=514.5$ incident at $\theta_{i}=70^{\circ}$ to the sample surface's normal. Further, surface Brillouin scattering showed an un-usual behaviour where the $\mathrm{L}_{K B 2}$ peak disappeared after some time of collection. This was attributed to 'wipe out' phenomenon where multiple reflections resulted in multiple inelastically scattered light being detected in the recrystallized layer.
\end{abstract}




\section{Introduction}

Ion implantation causes stresses and strains in the implanted region of a crystalline material due to the breaking of bonds along the damage cascade caused by the implanted ions by electronic and nuclear stopping processes. Healing of the stressed/ strained material is achieved by moving the target displaced atoms and implanted ion species to sites where the material attains minimum energy [1]. This is accomplished through post implantation thermal annealing. During annealing sufficient thermal energy is provided in order to recover the crystal structure of the material which was damaged in the collision cascades within the implanted regio i provided the defect density is not too high.

Diamond, with an electronic configuration $s p^{3}$ tends tr $f_{1} \ldots s p^{2}$ carbon during irradiation once a critical radiation damage is exceeded. For moderate $\mathrm{k}^{\mathrm{r}}$ implantation energies, typically used in semiconductor doping, the simulated threshold c am، ge density as obtained from Stopping and Range of Ions in Matter (SRIM) is $\approx 5-9 \times 10^{22} \mathrm{rac} / \mathrm{c}_{\mathrm{in}}{ }^{3}$ [2]. Other scientists, using $1 \mathrm{MeV}$ energies found the threshold for amorphization $\cdots$ dimulond to be $\approx 6 \pm 2 \times 10^{22} \mathrm{vac} / \mathrm{cm}^{3}$ [3]. Upon annealing, implanted diamond remains as ar amorphous $s p^{2} / s p^{3}$ matrix unlike other covalently bonded semiconductors $\mathrm{Si}$ and Ge that indergoes solid phase epitaxial regrowth [4]. It has been observed that for implantation above th = ritical damage density, the damaged layer graphitizes upon annealing and forms sharp $h$ sun ${ }^{\prime}$ aries with the diamond [5]. In a CIRA study using carbon ions and multiple energies, and $1 、 r$ a total fluence above the critical damage density, a uniformly damaged graphitic layer frol. th: surface to a depth of $\approx 0.25 \mu \mathrm{m}$ was created [6] and in that work we were able to $\mathrm{C}^{\prime} \mathrm{ta}^{\mathrm{n}} \mathrm{t}^{\mathrm{l}} \mathrm{e}$ e elastic properties of the amorphous carbon layer that was on top of the bulk diamond. This w srk sought to find out if a Rayleigh wave could be detected from the diamond layer above the graphitic layer. This was done by reducing the scattering volume to a level where the bulk modes $\mathrm{T}_{K B 2}$ and $\mathrm{L}_{K B 2}$ can just be observed in the thin diamond layer above the opaque amorphous layer. When this is achieved, the thin diamond layer can then be implanted with say carbon ions and the elastic properties of this layer could be studied using SBS as a function of ion fluence and annealing temperature below the damage threshold that causes graphitization.

In our previous work [6], one of the studies created a buried amorphous layer of $\approx 50 \mathrm{~nm}$ of recrystallized diamond on top of an amorphous carbon. In the work described in [6] the carbon ion-implanted diamond was annealed from $600-1200{ }^{\circ} \mathrm{C}$ and the changes were elucidated as 
observed by Raman spectroscopy (RS), surface Brillouin scattering (SBS), high resolution transmission electron microscopy (HRTEM), electron energy loss spectroscopy (EELS). In this paper we investigate SBS effects where the scattering volume of the undamaged diamond cap at the surface has been increased by implanting a lighter ion at a higher energy so that the implanted fluence generates an amorphous carbon layer deeper below the diamond surface.

\section{Experimental}

Four CVD diamond samples purchased from Element Six Comp ``y, UK, were $\mathrm{He}^{+}$implanted. Two of the diamond samples had one side (P1) unpolished whil : th other two were polished on both sides (P2). One P1 and one P2 was implanted with $50 \mathrm{~K} \cdot \mathrm{V} \mathrm{He}^{+}$and the remaining two samples was implanted at $160 \mathrm{keV} \mathrm{He}^{+}$. The P1 sampl s were implanted at liquid nitrogen temperature while the $\mathrm{P} 2$ 's at room temperature. The sa' ne fluence of $6 \times 10^{16} \mathrm{ions} / \mathrm{cm}^{2}$ was implanted in the four samples. The two samples im slr.,ted at liquid nitrogen were transferred from the cold stage end station target holder of the imp. anter into a liquid nitrogen bath and dropped into a preheated RF furnace at $1200{ }^{\circ} \mathrm{C}$ in flown ${ }^{\circ}$ argon gas. This process is called Cold Implantation Rapid Annealing (CIRA) was developec 'yy Prins to dope diamond [7]. The 2 samples implanted at RT were also annealed at $1200{ }^{\circ} \mathrm{C}$ n n . ving argon. The anneal time was one hour for each sample. The implantation procede e was similar to that explained in the experimental section of the paper by Motochi et al [6]. Hu vever, the furnace used for annealing was described in [8]. The resultant sample after this nroies, may be schematically visualized as shown in figure 1.

Figure 1: Cross-section 'f the He ion implanted diamond after annealing (not drawn to scale).

After annealing, the damage was investigated using Raman spectroscopy. Reflectivity studies using UV-VIS-NIR 500 Cary spectrophotometer was done to observe interference effects above the damaged region. Brillouin surface scattering was done to study surface acoustic waves in the thin film.

\section{$3 \quad$ Results and discussion}




\subsection{Stopping and Range of Ions in Matter (SRIM) simulation of $\mathrm{He}^{+}$ implanted in diamond}

A SRIM monte Carlo simulation for $\mathrm{He}^{+}$implanted in diamond is shown in Figure 2. The fluence of $6 \times 10^{16}$ ions $/ \mathrm{cm}^{2}$ used in this work creates a simulated damage profile where the TRIM damage density $\left(D_{c}\right)$ is above and below TRIM damage damage density threshold of $\left(4 \times 10^{22}\right.$ ions $\left./ \mathrm{cm}^{3}\right)[9$, 10] for helium ions in diamond. This implantation would result in a relatively undamaged near surface region. The region below $\mathrm{D}_{c}$ reconstruct by recombination of vacancies and interstials and not by solid state epitaxial growth from the boundary outwards, this explains the formation of sharp boundaries at the interfaces between the top layer, the amor snc 's carbon layer and the virgin diamond beyond the implantation region. The top region ela ns the diamond structure after annealing as the damage density within this volume is 6. low the critical damage density thus resulting in a thin layer of diamond sitting on an opaque zyes of amorphous carbon and beyond the amorphous layer is the diamond substrate which wan not aflected by the ions.

Figure 2: TRIM simulation for $80 \mathrm{keV}$ anc $16 \mathrm{KeV} \mathrm{He}^{+}$energies used in implantation in diamond.

As predicted by SRIM si $\mathrm{It}^{1}{ }^{1}$ aion, the $\mathrm{He}^{+}$implanted and annealed system is a thin transparent recrystallized diamind $1.1 \mathrm{~m} \approx 79 \mathrm{~nm}$ for $80 \mathrm{keV}$ and $234 \mathrm{~nm}$ for $160 \mathrm{keV}$ on an opaque amorphized layer oc thic nness $231 \mathrm{~nm}$ and $250 \mathrm{~nm}$ for energies $80 \mathrm{keV}$ and $160 \mathrm{keV}$, respectively. The amnrp ous layer with a sharp boundary at the end of range sits on pristine diamond at $\approx 310 \mathrm{~nm}$ al $1 \approx 484 \mathrm{~nm}$ for the $80 \mathrm{keV}$ and $160 \mathrm{keV}$ implants, respectively (see figure 2).

\subsection{Raman spectroscopy observations}

The T64000 Raman spectrometer using single line modes of $514.5 \mathrm{~nm}$ and $785 \mathrm{~nm}$ was used in measurements on the He ion implanted and annealed diamond samples. The measurement show the presence of two broad bands after annealing at $1200{ }^{\circ} \mathrm{C}$. The $\mathrm{D}$ peak usually centered around $1350 \mathrm{~cm}^{-1}$ is convoluted under a broad band that extends from $1100-1500 \mathrm{~cm}^{-1}$. A range of Gaussian curves was used to fit the shape of the recorded Raman spectra. The fit centered around 
the $\mathrm{G}$ band is more defined and lies between $1603-1605 \mathrm{~cm}^{-1}$ for both 514.5 and $785 \mathrm{~nm}$ light beams. There is therefore no dispersion in the $\mathrm{G}$ peak which implies that the opaque implanted layer has some graphitic order. This kind of spectra is indicative of the nature of an amorphous carbon layer and peaks around 1340-1350 are due to the D peak, while peaks around 1550-1650 is related to the G peak [11].

Figure 3: Raman spectra of the P1 He ion implanted diamond after annealing $1200{ }^{\circ} \mathrm{C}$. Figures (a) and (c) were implanted at $160 \mathrm{keV}$ while (b) and (d) were implanted at $80 \mathrm{keV}$. Light of wavelength $514.5 \mathrm{~nm}$ was used to produce spectra (a) and (b) while hat of wavelength $785 \mathrm{~nm}$ produced (c) and (d).

The presence of a broad D peak implies disorde : $n$ :e ion damaged region. However, a

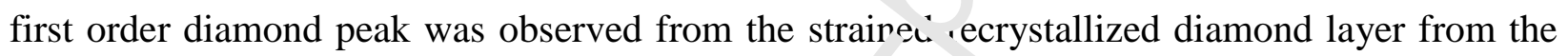
surface ( $\approx 79 \mathrm{~nm}$ for the $80 \mathrm{keV}$ implanted diamo id, that remained intact after annealing because the damage density was below the critical $\therefore n_{s}{ }^{\prime} y$ for amorphization. The first order diamond signal was observed around 1326/1327 $\mathrm{c}_{1}{ }^{-1}$ using the $514.5 \mathrm{~nm}$ light in figures $3 \mathrm{a}$ and $3 \mathrm{~b}$ implying that the diamond is under tens1s stress [12]. The diamond line is not apparent in the 785 $\mathrm{nm}$ spectra since the red light is more sf $\mathrm{n}$ i icive to non diamond phases.

\subsection{Transmission and cifluse reflectivity measurements}

The transmission spectra or $>2$ an unimplanted and as-implanted diamonds are shown in figure 4. Pure diamond transmittei $\approx 70 \%$ while the as-implanted transmitted $\approx 62 \%$ in the visible range. Transmission measurements conducted on all the samples after implantation and annealing show a less than $0.3 \%$ transmission of $514.5 \mathrm{~nm}$ light. This implies that the damaged region becomes opaque after annealing.

Figure 4: Transmission spectra in the visible light range of un-implanted CVD diamond, After it is implanted with $\mathrm{He}^{+}$at a fluence of $6 \times 10^{16}$ ions $/ \mathrm{cm}^{2}$ and the same sample after annealing at 1200 ${ }^{\circ} \mathrm{C}$. There is no transmission below $220 \mathrm{~nm}$, the absorption edge of diamond.

Constant angle reflection interference spectroscopy (CATIS) measurements in the visible 
range was conducted to determine the presence of a thin transparent medium sitting on an opaque surface. Figure 5 shows the diffuse reflectivity graphs of different surfaces measured using the UV-VIS-NIR 500 Cary spectrophotometer. It was observed that those surfaces with thin transparent diamond on an opaque graphitic layer (He-ion implanted samples) resulted in a sine-like curve [13] due to interference of waves reflected from the surface and the opaque surface at the first boundary of the diamond/opaque interface.

The 'wavelength' of the sine curve reduced with the wavelength of the light. The wavelength and amplitude of the $80 \mathrm{keV}$ implanted diamonds were relatively the same for the same interval change. The amplitude of $160 \mathrm{keV}$ implanted was smar' ${ }^{2}$ than the $80 \mathrm{keV}$ implanted diamonds by $49.4 \%$. Thick samples like pristine diamond or mea ure nents done at the back of the amorphous layer for P2 samples do not show any interferen $\iota_{\epsilon}$ frin yes. This is attributed to the fact that the width between the end of range of ions and th - havk surface (region labelled 'pristine diamond' in figure 1) was infinitely large compared to $i$ - the wavelength of light to show any interference phenomena.

Figure 5: Diffuse reflectance measurements $n$ the $\mathrm{P} 2$ samples and one $\mathrm{P} 1$ samples, front means the implanted side, RT is room temperatı "e implanted while CIRA were implanted at liquid nitrogen temperature. Analysis of wa $e^{\prime} e r$ th and amplitudes were done in the section labelled A. The line $\mathrm{B}$ is an instrumental arteí. ct

The thickness of th $\mathrm{ca}_{\mathbf{1}}$ ' 'ayer was estimated from the period of the interference pattern at normal incidence using [ 14 ].

$$
d_{k}=\left|\frac{1}{2 \eta\left(\frac{1}{\lambda_{i}}-\frac{1}{\lambda_{j}}\right)}\right|
$$

where $d_{k}$ is the layer thickness, $\eta$ is the refractive index of the diamond, and $\lambda_{i, j}$ are the wavelengths corresponding to two consecutive maxima (or minima) in the interference pattern. The maxima and minima values of the curves are shown in table 1

Table 1: Trough and crest positions from interference pattern observed on thin diamond cap after annealing He ion implanted diamond 


\begin{tabular}{|l|l|l|l|l|l|}
\hline Implantation & Implantation \\
environment & energy $(\mathrm{keV})$ & Feature & \multicolumn{3}{|l|}{ Peak position $(\mathrm{nm})$} \\
\cline { 3 - 6 } & 80 & & 1 & 2 & 3 \\
\hline Room temp. & & Trough & 283 & 239 & 212 \\
\cline { 3 - 7 } & \multirow{2}{*}{ CIRA } & Crest & 313 & 257 & 223 \\
\cline { 3 - 7 } & & Trough & 328 & 260 & 222 \\
\cline { 3 - 7 } & 160 & Crest & 289 & 238 & 209 \\
\hline & & Trough & 278 & 231 & 207 \\
\cline { 3 - 7 } & & Crest & 311 & 252 & 220 \\
\hline
\end{tabular}

The incident radiation normal to the surface changes from ne $r$ infrared to UV. Equation 1 is modified to account for the continuously changing wavel $\mathrm{en}_{\mathrm{e}}^{\text {th }}$ of the incident radiation to:

$$
d_{k 1}=\frac{1}{2 \eta\left(\frac{1}{\lambda_{3}}-\frac{1}{\lambda_{2}}\right)}-\frac{1}{2 \eta\left(-\frac{1}{2}-\frac{1}{7}\right)}
$$

The average from the trough and crests of he same curve gives the estimated cap thickness, thus:

$$
\left.d=\frac{1}{\Delta_{\ddots_{1}} \mid} \frac{1}{\left(\lambda_{3}^{1}-\frac{1}{\lambda_{2}}\right)}-\frac{1}{\left(\frac{1}{\lambda_{2}}-\frac{1}{\lambda_{1}}\right)}\right]
$$

Taking the refractive inde. 0 . aramond as 2.5 around the $300 \mathrm{~nm}$ value [15] and using equation (3), the estimated thich ess of the recrystalized diamond cap were $\approx 58 \mathrm{~nm}, 63 \mathrm{~nm}$ for the room temperature and $\mathrm{Ci}^{2} \mathrm{~A} I$ elium implanted at $80 \mathrm{keV}$, respectively. For the CIRA, $160 \mathrm{keV}$ implanted diamond, the c ap $\mathrm{t}$ ickness was calculated to be $\approx 103 \mathrm{~nm}$. These values differ from those theoretically calcula ed from SRIM. It is likely that the critical damage density is lower for implanted layers that are close to the diamond surface. It has been observed that for high energies ¿1 MeV, the critical damage density to achieve amorphization is higher [3].

\subsubsection{Reflection from diamond surface and buried graphitic layer}

As predicted by the TRIM simulation, (figure 2) and confirmed by calculations in section 3.3, helium implantation at single energy resulted in a damaged layer buried beneath a diamond layer after annealing at $1200^{\circ} \mathrm{C}$. The interface of the undamaged diamond cap and buried thin film of graphitic carbon forms a mirror-like surface with a reflective surface. 
When light is incident onto the top surface of the diamond cap, the incident ray is partly reflected and partly transmitted as refracted light. The transmitted ray, upon arrival at the interface gets partly reflected towards the surface while some fraction of it is absorbed. At the 'top' surface, some light is transmitted while some gets reflected back towards the interface. The transmitted light moves in the same direction as that initially reflected from the incident light, though with a longer pathlength. The reflected light repeats the process leading to multiple reflections. The intensity of the multiple reflections reduces after each refection. This process goes on until the light reaches the edge of the diamond film as shown in figure 6.

Figure 6: Multiple reflection of light on the thin diamond film s. tins, on He ion damaged region

No light goes through the amorphized layer but $e_{\ldots}=\eta_{i}{ }^{c}$ some were to go through, it would be diffusely reflected at the unpolished surface and neers $v$ further analysis.

The intensity of the reflected beam can th ${ }_{\mathrm{s}}$ be calculated from Fresnel's equations. Reflecting power or reflection coefficient ( $P$; is ' e ratio of the reflected to incident intensity [16].

The complex refractive index $n=n_{r}(1-i \chi)$

for $\chi=1$

$$
R=\frac{\left(n_{r}-1\right)^{2}}{\left(n_{r}+1\right)^{2}} \cdot \frac{{r^{2}}^{2}}{\eta_{r}^{2}}=\frac{2 n_{r}^{2}-2 n_{r}+1}{2 n_{r}^{2}+2 n_{r}+1}=\frac{1-1 / n_{r}}{1+1 / n_{r}} \approx 1-\frac{2}{n_{r}}
$$

For a non transmitting $\mathrm{m}$. dium, absorption $(A)=1-R$

$$
A=1-R=\frac{4 n_{r}}{2 n_{r}^{2}+2 n_{r}+1} \approx \frac{2}{n_{r}}
$$

If the $\mathrm{He}^{+}$implanted layer is more graphitic after annealing then from [17], the refractive index for $500 \mathrm{~nm}$ light at room temperature is $\approx 2.6$, hence; $R=0.23$ and $A=0.77$.

$$
R=\frac{(n-1)^{2}}{(n+1)^{2}} \Rightarrow \frac{\left(\frac{n_{2}}{n_{1}}-1\right)^{2}}{\left(\frac{n_{2}}{n_{1}}+1\right)^{2}}=\frac{\left(n_{2}-n_{1}\right)^{2}}{\left(n_{2}+n_{1}\right)^{2}}
$$

For light incident from air or vacuum, $n_{2} \rightarrow 1$, and letting $n_{1} \rightarrow n$, the refractive index of diamond.

$$
R=\left(\frac{1-n}{1+n}\right)^{2}
$$




$$
R_{1 \rightarrow n}=R_{n \rightarrow 1} \text {, similarly } T_{1 \rightarrow n}=T_{n \rightarrow 1}
$$

Supposing the medium does 'not absorb' any light then $T=(1-R)$.

The total reflectance is therefore,

$$
\begin{aligned}
& R_{\text {Total }}=R+T^{2} R+T^{2} R^{3}+T^{2} R^{5}+\cdots+T^{2} R^{\infty} \\
& =R+T^{2} R\left\{1+R^{2}+R^{4} \cdots+R^{\infty-1}\right\} \\
& =R+(1-R)^{2} R\left\{1+R^{2}+R^{4} \cdots+R^{\infty}\right\}
\end{aligned}
$$

Expanding the series in \{\} brackets we obtain the total reflectivity.

$$
R_{\text {Total }}=R+\frac{(1-R)^{2} R}{1-R^{2}}=\frac{2 R-2 R^{2}}{1-R^{2}}=\frac{2 R(1-R}{(1-R)(1+R)}=\frac{2 R}{1+R}
$$

For $R=0.23$, then $R_{\text {Total }}=0.37$ of the intensity of nc de .t beam. This implies that light intensity that reaches the detector is very small hence thə lo $\mathrm{g}$ time required to collect spectra.

\subsection{Surface Brillouin scattering resv ts}

The $\mathrm{He}$ ion implanted and annealed syste $\mathrm{i}_{\mathrm{i}} \mathrm{w}_{\mathrm{A}} \mathrm{S}$ a thin transparent diamond film $\approx 63 \mathrm{~nm}$ for 80 $\mathrm{keV}$ and $103 \mathrm{~nm}$ for $160 \mathrm{keV}$, CIRA to ated samples as calculated from equation (3). This work sought to investigate the surface Brill $\mathrm{u}_{\llcorner}$acoustic modes in the recrystalized diamond cap sitting on the amorphous carbon. The amc rpı nus layer which has a sharp boundary at end of range sits on pristine diamond. The top diamı nd layer on the amorphized layer is basically an optically hard material on a soft one. The extected behaviour of such a system for opaque samples is the observation of a Rayl igh. mo le of the top layer only [18]. However, the transparent nature of the thin diamond film therefo e means the scattering volume and film-substrate interface properties has the potential to alter the outcome.

SBS measurements were done on P1 samples. The amorphous layer is opaque with a transmission of less than $0.25 \%$ hence there is no transmission through it. However, If some light passes through the layer, there can be no spectrum obtained from the unpolished surface due to diffuse scattering and any light specularly reflected back ends up being completely absorbed in the layer. The experimental setup using P1 samples of figure 6 therefore guarantees no light from the back of the layer. The spectra observed to be emanating from indirect scattering must be from the diamond sitting on top of the graphitic layer which was found to have reflective properties (see 
section 3.3). The scattering geometry which applies for transparent sample shown in figure 7 was employed for analysis [19].

Figure 7: Scattering geometry which best represents a transparent sample like diamond. Optical wavevectors are represented by thin arrow lines while thick arrows show acoustic wavevectors of bulk $\left(\boldsymbol{k}_{b}\right)$ and surface $\left(\boldsymbol{k}_{s}\right)$ acoustic waves. $\Omega_{i}, \Omega_{s}$ are the incident and scattered optical angular frequencies; $\omega$ is the acoustical angular frequency; $\mathrm{q}_{s}, \mathrm{q}_{s}$ are the incident and scattered optical wave vectors in 'vacuum'; $\boldsymbol{q}_{i}^{\prime}, \boldsymbol{q}_{s}^{\prime}, \boldsymbol{q}_{i}^{\prime \prime}, \boldsymbol{q}_{s}^{\prime \prime}$ are the incident and $\mathrm{r}$ flected optical wave vectors inside the medium before and after reflection, respectively, $\theta$ is he :ncident and scattering angles of the optical wave vector in $\square$ 'vacuum' while $\theta^{\prime}$ is the refr 4 cte $t$ and reflected angles of the acoustical wave vector at the sample holder [20]

Measurements involving an extended free su tral range (FSR) produced $\mathrm{L}_{K B 1}$ after a very long collection time as expected due to the limite 1 scuicering volume of the top thin diamond layer. The longitudinal peak $\mathrm{L}_{K B 1}$ at $\approx 42 \mathrm{GH}_{\bar{c}} \mathrm{~d}_{\mathrm{j}}$ - to direct scattering was therefore from strained diamond sitting on the opaque layer, fo: laser beam $\lambda=514.5$ incident at $\theta_{i}=70^{\circ}$ to the sample surface's normal [19].

Weak peaks were observe' in positions $\mathrm{L}_{K B 2}$ and $\mathrm{T}_{K B 2}$ using p-polarized and s-polarized light, respectively. These peak w vuld however disappear (a phenomenon we called 'wipe out') as measurements progressed. Th in directly scattered $\mathrm{L}_{K B 2}$ peak observed after close to 4 hours seems to have been complett: $v$, ine $d$ out after 20 hours but seems to be growing again after 34 hours as seen in figure 8.

Figure 8: Representative SBS spectra collected at the front of a $160 \mathrm{keV}$ CIRA treated sample, showing cumulative acquiring up to 34 hours, showing indirectly scattered $\mathrm{L}_{K B 2}$ mode at $\approx 61.6$ $\mathrm{GHz}$.

Beghi et al [20] assert that in indirect backscattering (also called $2 \alpha \mathrm{A}$ and only applicable to transparent materials), bulk acoustic modes are probed if the geometric wavelength $\left(\lambda^{\prime}\right)$ is shorter than the film thickness. If however, the film thickness is smaller than the geometric 
wavelength of the probing light, other surface waves like Sezawa waves may be observed. In this section, where it is intended to get light reflect off the deep-seated 'mirror' and back through the diamond, the geometric wavelength of the layer would be important. In such a configuration the geometric wavelength $\lambda^{\prime}=\lambda_{o} / 2 \sin \theta$, is independent of the refractive index of the transparent film. Thus for angles of incidence that vary between $30^{\circ}$ and $80^{\circ}$, the film thickness needs to be greater than $514.5 \mathrm{~nm}$ and $261.2 \mathrm{~nm}$ respectively, to observe the bulk waves due to indirect scattering. The bulk waves were observed in 0.3-0.5 mm thick samples as reported by Motochi et al [19]. Similarly, Beghi et al [20] studying bulk silica (2 mm thi $=\mathrm{k})$ and thin silica film (2 $\mu \mathrm{m}$ thick) were able to observe a weak Rayleigh peak in bulk silir $\mathrm{us}_{\mathrm{ing}}$ backscattering ( $2 \alpha \mathrm{A}$ ) geometry but they do not report observing it in the forward $(\mathrm{O} \cap \mathrm{P}, \ldots$ transmission $(90 \mathrm{~A} / \mathrm{platelet})$ geometries on the bulk or thin film samples. In their large $\operatorname{ar}_{\mathbf{r}_{\mathbf{r}}} \cdot \mathrm{es}$ they were able to observe the bulk modes (transverse and longitudinal). Therefore, rot ing a thin transparent diamond on a reflective opaque layer using the backscattering $(2 \alpha /)$ ge गmetry in this work was appropriate. Using P2 sample implanted at $160 \mathrm{keV}$, the large $\mathrm{stt}$ ring volume at the back of the layer easily yields the bulk modes as shown in figure $9 \mathrm{Wr}$ en lie same sample is turned so that light impinges from the front (small scattering volume), no obvious peaks were observed. Pronounced longitudinal modes from the back of the : ayer were observed $\mathrm{L}_{K B 2}$ at $64.2 \mathrm{GHz}$ while similar modes from the front $\mathrm{L}_{K B 2}$ at $61.6 \bumpeq \mathrm{H}_{\llcorner}$were observed for laser beam $\lambda=514.5$ incident at $\theta_{i}$ $=70^{\circ}$ to the sample surface's no, nal. The difference is due to strain in the diamond film above the amorphous layer. This corro:'orat s the results of the Raman measurement in section 3.2 where the diamond signal was rbsc rvec around 1326/1327 $\mathrm{cm}^{-1}$ instead of the Raman shift characteristic value for diamond, $1332 \mathrm{c}^{-1}[21]$.

Figure 9: SBS spectra collected at the front and back of a P2, $160 \mathrm{keV}$ room temperature He ion implanted sample. There were small peaks, $\mathrm{L}_{K B 2}$ at $61.6 \mathrm{GHz}$ observed for the measurements from the front (red spectra), compared to measurement from the back (black spectra) that yielded pronounced indirectly scattered peaks $\mathrm{T}_{K B 2}$ at $46.5 \mathrm{GHz}$ and $\mathrm{L}_{K B 2}$ at $64.2 \mathrm{GHz}$ for laser beam $\lambda=514.5$ incident at $\theta_{i}=70^{\circ}$ to the sample surface's normal.

The 'wipe-out' phenomenon was attributed to multiple reflections leading to what can be 
perceived as multiple sources of coherent inelastically scattered light being detected. Interference effects by division of the light would be expected when light is incident at an angle on such surfaces (two reflecting surfaces). In this scenario, part of the light is reflected from the top surface and the other is reflected at the interface. When these reflected beams overlap, interference effects (contour fringes) are observed due to the path difference traveled by light in the thin layer as shown in figure 6 , the optical path difference is $2 n t \cos \theta$, where $n$ is the refractive index of the medium between the reflecting surfaces, $t$ is the thickness of the layer and $\theta$ is the angle made with the normal by the light path in the medium. The approximate intensity of light detected due to multiple reflections between $\approx 100 \%$ reflective "surfaces" is calcuinted in section 3.3.1. From these calculations the ideal calculated total reflectivity was $37 \% \mathrm{t}$ 't $t^{1}$ is could be much less owing to absorption of the layer on the implanted side. Which exr la. 'ns ' ne long time scales required for accumulation of photons scattering off the phonons to yien' the indirect scattered peaks that later undergo a 'wipe-out' effect.

\section{Conclusion}

This work sought to study bulk modes $\neg n$ recrystallized diamond after implanting with $\mathrm{He}^{+}$and annealing at $1200{ }^{\circ} \mathrm{C}$. The Raman a 10 ¿ . ffuse reflectance measurements show a recrystallized diamond film that forms a cap on th $\mathrm{e} b \mathrm{c}$-ried graphitic region. The diamond signal from the cap was observed around 1326/1327 $\mathrm{cm}^{-1}$ instead of the Raman shift characteristic value for diamond which is always observed at $1.32 \mathrm{~cm}^{-1}$ implying that the recrystallized diamond was strained. The thin recrystallized dia.no:d l yer was estimated to be $\approx 103 \mathrm{~nm}$ for implantation at $160 \mathrm{keV}$ using the constant angle eflection interference spectroscopy. SBS measurements on this cap produced weak modes $\mathrm{T}_{K B 2}$ at $42.2 \mathrm{GHz}$ and $\mathrm{L}_{K B 2}$ at $61.6 \mathrm{GHz}$ for laser beam $\lambda=514.5$ incident at $\theta_{i}=70^{\circ}$ to the sample surface's normal. However, the peaks suffered from 'wipe-out' effect. The 'wipe-out' was attributed to multiple scattering and interference effects arising from light reflecting off the diamond surface the diamond-graphitic interface buried below the thin diamond cap.

The support of the DST-NRF Centre of Excellence in Strong Materials (CoE-SM) towards this research is hereby acknowledged. Opinions expressed and conclusions arrived at, are those of the author and are not necessarily to be attributed to the CoE-SM. Special thanks to Dr. R. M. 
Erasmus for useful consultations and for the Raman spectroscopy measurements.

\section{References}

[1] R. A. Spits, T. E. Derry, J. F. Prins 1992, Annealing studies in ion implanted diamond, Nucl Instr. and Meth. B, 64,210-214

[2] J. F. Ziegler and J. P. Biersack and M. D. Ziegler 2008, The stopping and range of ions in matter, Publisher: SRIM Co. Chester, Maryland, pages, 2-1

[3] D. P. Hickey, K. S. Jones and R. G. Elliman, 2009 Amorph: ation and graphitization of single crystal diamond -A transmission electron microsc py . tudy, Diam. Relat. Mat. 18, $1353-1359$

[4] J. O. Orwa, K. W. Nugent, D. N. Jamieson and S. F. ' $w e r$ 2000, Raman investigation of damage by deep ion implantation in diamond, F. vs. Rev. B, 62 5461-5472

[5] T. E. Derry, E. K. Nshingabigwi, M. Levitt s Neething, and S. R. Naidoo, 2009 Cross-section transmission electron mic oscupy of the ion implantation damage in annealed diamond, Nucl. Instr. anc. M ch. B, 267, 2705-2707

[6] I. Motochi, S. R. Naidoo, B. A. Mathe, R. Erasmus, E. Aradi, T. E. Derry, E. J. Olivier 2015 Surface Brillouin scatter in, >n annealed ion-implanted CVD diamond, Diam. Relat. Mat. 56, 6-12

[7] J. F. Prins and T. E. Dert, 20v0 Cross-section transmission electron microscopy of the ion implantation damage in arnealed diamond, Nucl. Instr. and Meth. in Phys. Res. B, 166-167, 364- 273

[8] I. Motochi, B. A. I 'athe, S. R. Naidoo, D. Wamwangi, T. E. Derry 2017 Surface Brillouin scattering observation of higher order resonances in annealed, ion-implanted CVD diamond, Diam. Relat. Mat. 76, 171-176

[9] C. Uzan-Saguy and C. Cytermann and R. Brener and V. Richter and M. Shaanan and R. Kalish 1995, Damage threshold for ion-beam induced graphitization of diamond, Appl. Phys. Lett. 67 1194-1196

[10] A. V. Khomich, R. A. Khmelnitskii, V. A. Dravin, A. A. Gippius, E. V. Zavedeevand and I. I. Vlasov 2006, Radiation damage in diamond subjected to Helium implantation, Appl. Phys. Solid. Stat. 49 1661-1665 
[11] A. C. Ferrari and J. Robertson, 2001, Resonant Raman spectroscopy of disordered, amorphous, and diamondlike carbon, Phys. Rev. B, 64, 075414-1

[12] Ingrid De Wolf, 1996, Micro-Raman spectroscopy to study local mechanical stress in silicon integrated circuits, Semicond. Sci. Technol, 11, 139-154

[13] A. M. Goodman, 1978, Optical intereference method for the approximate determination of refractive index and thickness of a transparent layer, Appl. Optics, 17, 2779-2787

[14] M. Born and E. Wolf, 2003, Principles of optics: Electromagnetic theory of propagation, interference and diffraction of light,7th Ed., pg 317-319

[15] G. Turri, S. Webster, Y. Chen, B. Wickham, A. Bennet and $\ldots$ Bass, 2017 Index of refraction from near ultra-violet to the near-infrared fror.' sir gle crystal microwave assisted CVD diamond, Optical Mat. Express. 7. No3, 85 -859

[16] D. W. Wheeler and R. J. K. Wood, 1999, Erosiv . ou behaviour of thick CVD diamond coatings, Wear. 225-229, 523-536

[17] B. J. Stagg and T. T. Charalampopoulos, 1 ,9', Refractive indices of pyrolytic graphite, amorphous carbon, and flame soot ir . $m_{\uparrow}$ ' arture range $25^{\circ} \mathrm{C}$ to $600^{\circ} \mathrm{C}$, Combusition and Flame. 94, 381-396

[18] C. Sumanya, J. D. Comins and A. F. Every, 2007 Surface Brillouin scattering in opaque thin films, J. Phys.:Conference Ser ıs 92, 012103

[19] I. Motochi, B. A. Mathe, S. R. Naidoo and T. E. Derry, 2016 Surface Brillouin scattering in ion-implanted chemica.' var 'or deposited diamond, Mat. Today: conference proceedings 3S, S145-S152

[20] M. G. Beghi, F. ग1 Fr .ızo, S. Pietralunga, C. Ubaldi and C. E. Bottani, 2011 Precision and accuracy in film " ffness measurements by brillouin spectroscopy, Rev. Sci. Instrum. 82, 0533107-(1-11)

[21] A. Dychalska, P. Popielarsrski, W. Frankow, K. Fabisiak, K. Parprocki, M. Szybowicz, 2015 Study of CVD diamond layers with amorphous carbon admixture by Raman scattering spectroscopy, Mat. Sci. Poland. 334, 799-805 


\section{Author Contribution}

I Motochi: Conceptualization, Methodology, Investigation, Formal Analysis, Writing original draft.

E. Aradi: Formal Analysis, Validation, Writing-Review and editing.

B. A. Mathe: Conceptualization, Supervision, Methodology.

S.R. Naidoo: Conceptualization, Resources, Writing-Review and editing, Supervision, Funding acquisition. 


\section{Journal Pre-proof}

Conflict of interest

There is no conflict of interest between the author and the funder 
Graphical abstract

\section{Highlights}

- Rapid annealing technique was used to recrystallize cold helium implanted diamond samples

- Analyses with Raman spectroscopy and diffuse reflectance spectroscopy indicated recrystallization of the samples.

- Wipe-out phenomenon not observed before led to un-usual presence of strained $\mathrm{SP}^{3}$ scattering in surface Brillouin. 


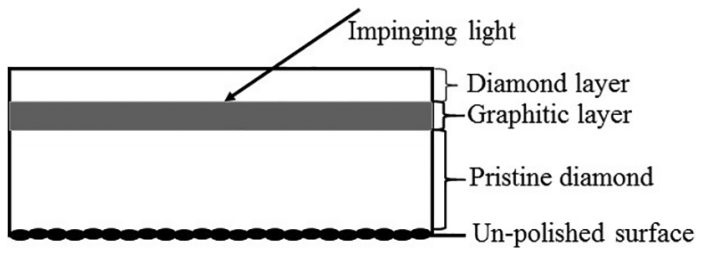

Figure 1 


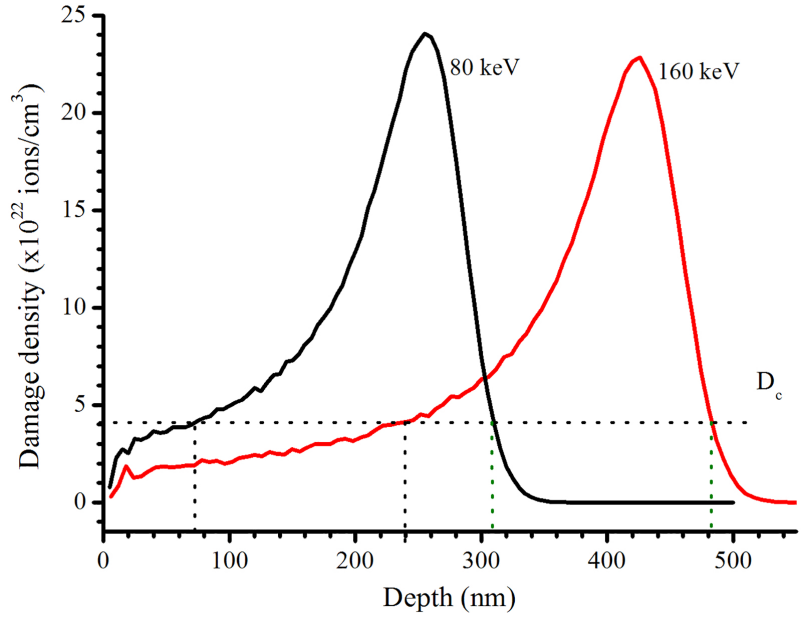

Figure 2 

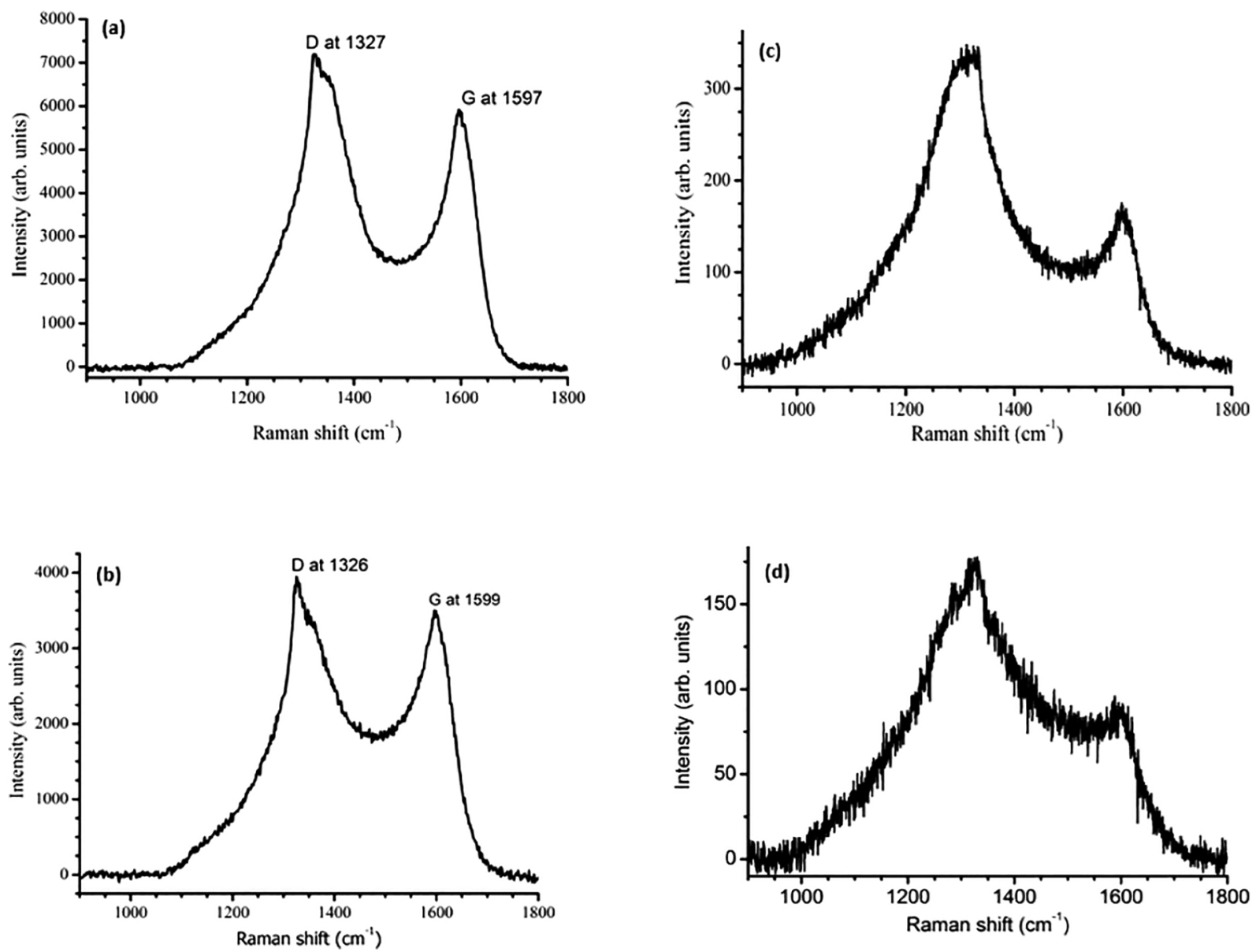

Figure 3 


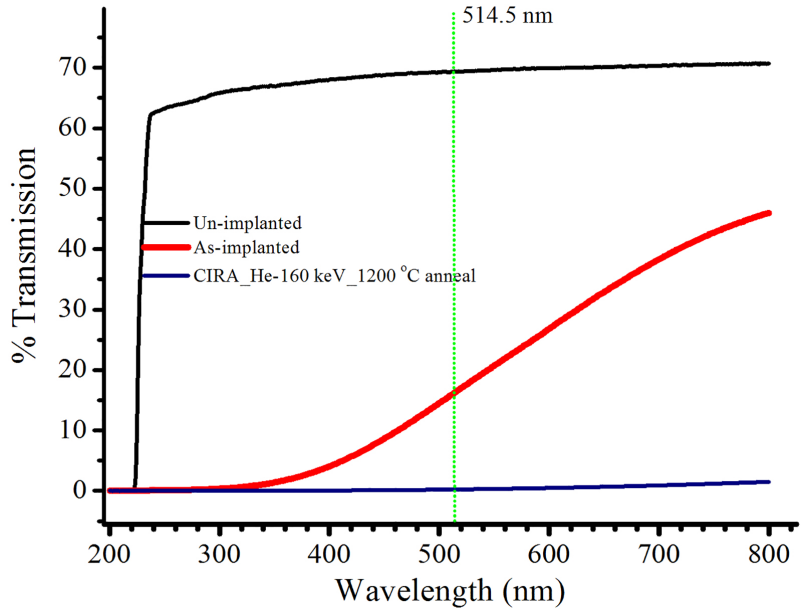

Figure 4 


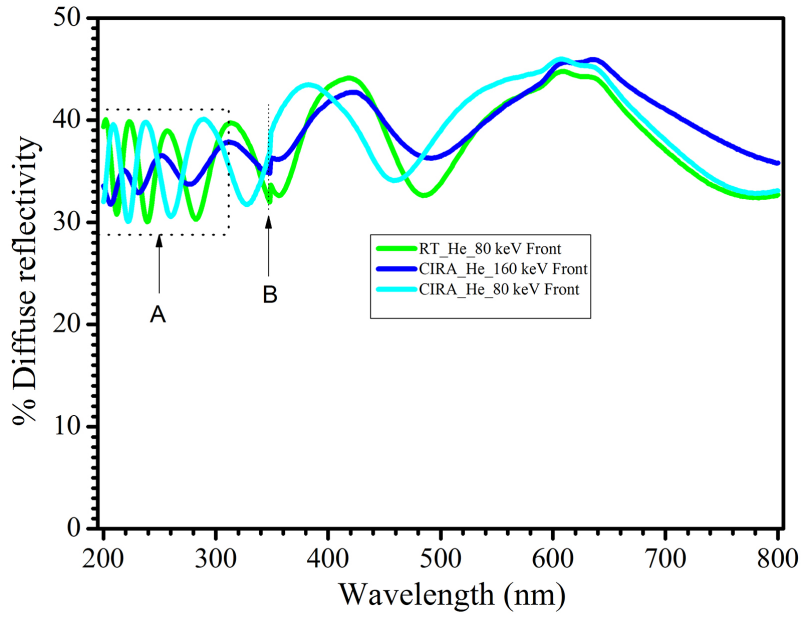

Figure 5 


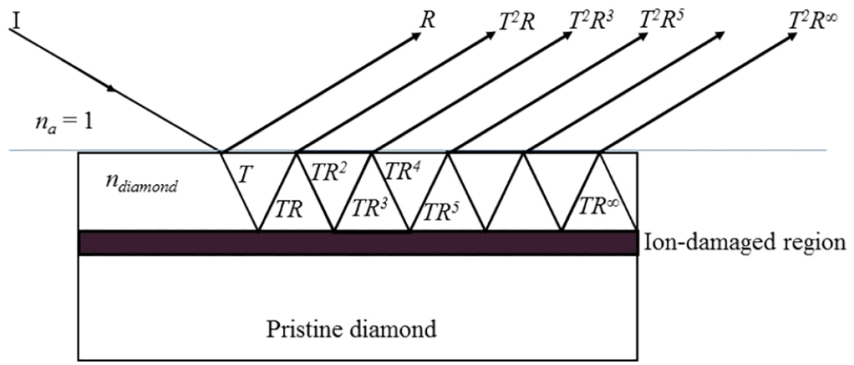

Figure 6 


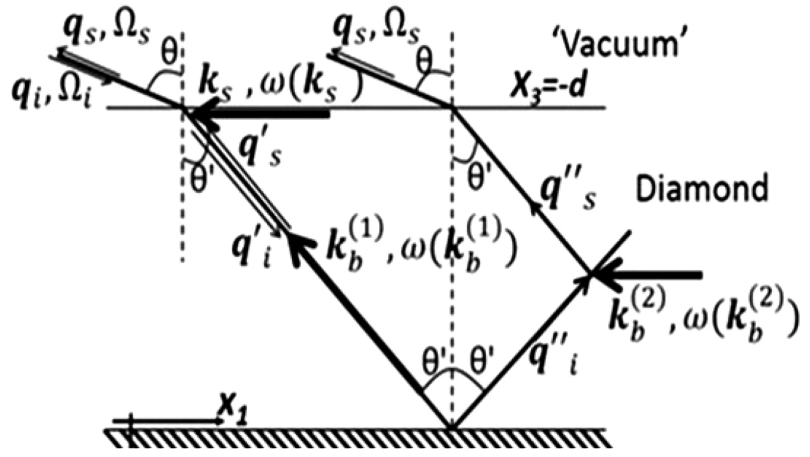

Figure 7 


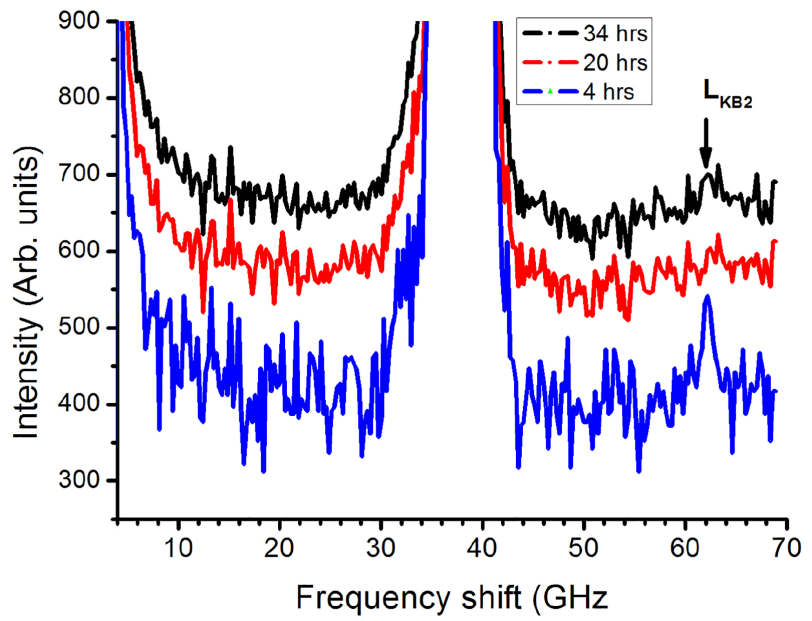

Figure 8 


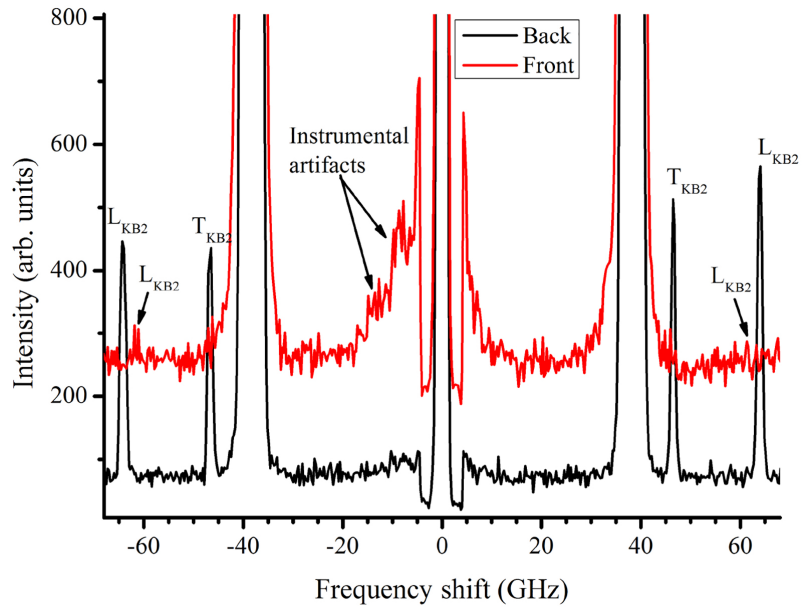

Figure 9 